\title{
Disparities in Quality of Primary Care by Resident and Staff Physicians-Reply
}

\author{
Utibe R. Essien, MD, MPH ${ }^{1,2}$ (1) and Steven J. Atlas, MD, MPH,
}

'Division of General Internal Medicine, University of Pittsburgh School of Medicine, Pittsburgh, PA, USA; ${ }^{2}$ Center for Health Equity Research and Promotion, VA Pittsburgh Healthcare System, Pittsburgh, PA, USA; ${ }^{3}$ Division of General Internal Medicine, Massachusetts General Hospital, Boston, MA, USA; ${ }^{4}$ Harvard Medical School, Boston, MA, USA.

J Gen Intern Med 35(3):939

DOI: $10.1007 / \mathrm{s} 11606-019-05347-2$

(c) Society of General Internal Medicine 2019

$\mathrm{W}$ e thank Dr. Weppner and colleagues for bringing to reader's attention a recent research letter that also examined outcomes of outpatient care by resident and staff physicians. In contrast to our study, that found significant differences in outcomes among primary care patients of resident and staff physicians in a single academic medical center, Edwards et al. found similar outcomes of care despite also demonstrating striking differences in baseline characteristics between patients seen by resident and staff physicians in the VA. Dr. Weppner speculates about the importance of systems of care developed by the VA that support the care of patients, regardless of whether their physician is a resident or attending. They also cite efforts in VA to better train resident physicians in team-based care and suggest that continuity of the team may overcome the limited continuity of care that resident physicians have with individual patients. Given that the mean number of years that a patient was in the VA system was 8.7 and 9.9 years for resident and attending physicians, respectively, it is likely that more sophisticated measures of teambased care are needed than simply quantifying the amount of time a patient has been followed by a resident physician.

Though it is possible that differences in study methods may account for some of the variation in outcomes observed between the two studies, these are unlikely to fully explain these disparate results. For instance, while we agree that a limitation of our study is the generalizability of our findings to all residency-based clinics, the recent VA study only examined outcomes of patients cared for in teaching primary care clinics, which likely underestimated the differences observed between resident and attending primary care physicians. Additionally, our study found similar differences across the domains of chronic disease management, preventive services, resource utilization, and patient experience of care, whereas the VA study examined a narrower set of domains. They also found variation among outcomes, such as similar results among resident and staff physicians for diabetes care and hospitalizations, but inferior outcomes for residents in hypertension management and use of high-risk medications in older patients. Whether these differences among outcomes examined reflect the underlying systems infrastructure or the care provided by resident and attending physicians was not explored.

Identifying strategies to provide high-quality and equitable care for patients in the primary care setting, including those seen by resident physicians, is vital to advancing health equity in vulnerable populations. As residency leaders balance the challenge between training and providing high-quality and equitable care, implementing the lessons learned from successful health systems may help reduce future disparities.

Corresponding Author: Utibe R. Essien, MD, MPH; Division of General Internal Medicine University of Pittsburgh School of Medicine, 3609 Forbes Avenue, Suite 2, Pittsburgh, PA 15213, USA (e-mail: uessien@pitt.edu).

\section{Compliance with Ethical Standards:}

Conflict of Interest: The authors declare that they do not have a conflict of interest.

Publisher's Note Springer Nature remains neutral with regard to jurisdictional claims in published maps and institutional affiliations. 